\title{
Actividades curriculares en necesidades educativas especiales de sujetos
}

\section{disléxicos \\ Curricular activities in special educational needs of dyslexic subjects}

\author{
María González Vázquez, Pilar Vieiro Iglesias, Mª Luisa Gómez Taibo y Teresa García \\ Universidade da Coruña
}

\begin{abstract}
Resumen
El objetivo de este trabajo es la aplicación de un programa de mejora de la lectura a nivel curricular con una alumna con DAL. Se ha realizado un estudio de caso a una niña de diez años con dificultades en la lectura. La intervención tuvo lugar en el centro escolar durante las sesiones de audición y lenguaje. Se le ha realizado una evaluación inicial en la que se determinó la necesidad de aplicar un programa en el que se trabajase la conciencia fonológica y la discriminación visual espacial. La instrucción consistió en el desarrollo de 10 sesiones de conciencia fonológica en las cuales se trabajó la conciencia de palabra, la conciencia silábica y el deletreo. Y finalmente, dos sesiones en las que se llevaron a cabo actividades de discriminación visual espacial con objetos, con figuras y con letras. Los resultados obtenidos después de la aplicación de este programa mostraron una mejoría en distintos aspectos relacionados con la lectura. Se consiguió mejorar su lectura en general, reduciendo el silabeo y mejorando su capacidad de deletreo. Palabras clave: Conciencia fonológica, aprendizaje de la lectura, discriminación visual espacial, intervención.
\end{abstract}

\section{Abstract}

The aim of this work is to implement a reading improvement program. A study case has been carried out on a ten years old girl with reading difficulties. The intervention took place in the school during the hearing and language sessions. An initial evaluation has been made in which the need to apply a program in which phonological awareness and visual discrimination are worked out. The instruction consisted in the development of 10 sessions of phonological awareness in which word consciousness, syllabic awareness and spelling were worked out. And finally, two sessions in which visual space discrimination was worked with objects, figures and letters. Results obtained after the application of this program showed an improvement in different aspects related to reading. It was possible to improve its reading in general, reducing the syllabus and improving its spelling ability

Key words: Phonological awareness, reading learning, spatial visual discrimination, intervention.

\section{Introducción}

La lectura es una tarea compleja en la cual intervienen muchos procesos cognitivos. Según Cuetos, Rodríguez, Ruano y Arribas (2012) lo primero que hacemos al leer es fijar las letras en nuestra fóvea para que la información llegué al área visual del cerebro. Nuestros ojos permanecen fijos en una palabra durante 200 milisegundos (fijaciones) y después realizan un pequeño salto para situarse en el trozo de texto siguiente. Este salto lo denominamos movimiento sacádico. Una vez nos fijamos en una palabra la analizamos visualmente, con lo cual ponemos en marcha el proceso de descodificación (transformación de palabras escritas en sonidos en la lectura en voz alta o en significados si se trata de lectura silenciosa). Para ello, identificamos las letras que componen las palabras y a continuación debemos reconocer la palabra y descifrar lo que representa cada grupo de letras.

A través de dos vías llegamos a la representación léxica de la palabra. Cada una de estas vías se emplea en diferentes casos (Juzmán, 1997).

Una de ellas es la "vía léxica" o "ruta visual" que consiste en comparar la forma ortográfica de la palabra con una serie de representaciones almacenadas en nuestra memoria. Para emplear esta ruta, lo único que necesita un lector es un almacén de léxico mental donde se encuentran representadas todas las palabras conocidas. Una vez que este lector identifica la palabra es necesario acudir al sistema semántico para saber a qué concepto representa. Sin embrago, mediante esta vía solo podemos leer las palabras que son conocidas ya que las palabras desconocidas o las pseudopalabras no están representadas en nuestra memoria a largo plazo (Juzmán 1997; Vieiro, Peralbo y García, 1997).

La ruta de acceso al léxico es la fonológica, también conocida como vía subléxica. Esta consiste en identificar las letras que componen cada palabra para después recuperar los sonidos que corresponden a esas letras mediante un mecanismo denominado conversión de grafema a fonema. Es decir, transformando cada grafema en su correspondiente sonido o fonema y leyéndolo en voz alta. De este modo el lector accede a la pronunciación de las palabras para luego recuperar de su léxico auditivo la representación de los sonidos (Vieiro, Peralbo y García, 1997).

Para llegar al significado de las palabras, la vía léxica es la más rápida y directa ya que, por la vía subléxica, tenemos que hacer uso del lenguaje oral para llegar al 
significado. Sin embargo, a la hora de leer debemos combinar adecuadamente ambas vías. Para leer una palabra desconocida la única forma es transformando los grafemas en fonemas (vía subléxica), pero si leemos una palabra conocida la mejor ruta es la léxica ya que, al conocer la palabra podemos recuperar la pronunciación global (Cuetos, Rodríguez, Ruano y Arribas, 2012).

Sin embargo, cuando leemos un texto con el fin de comprender lo que está escrito no es suficiente con descodificar los signos y letras, reconocer las palabras y llegar a su significado. Para poder llegar a comprender el texto son necesarios los procesos sintácticos y semánticos (García, 1993) a los cuales no dedicaremos una disertación teórica sobre su funcionamiento ya que no son objeto de estudio de esta investigación.

Existen una serie de habilidades que nos permiten comenzar a leer. Una de estas habilidades es la conciencia fonológica.

Según Ripoll y Aguado (2015) la conciencia fonológica son los conocimientos acerca de los sonidos del habla y sus combinaciones. Otros autores la definen como una habilidad metalingüística que consiste en segmentar los componentes del habla en unidades. Así, podemos dividir un discurso en oraciones, las oraciones en palabras, las palabras en sílabas y las sílabas en fonemas. El fonema se considera la unidad más básica que se puede aislar. Un buen lector debe conocer las correspondencias entre grafemas y fonemas, es decir, entre las letras y los sonidos. (Ripoll y Aguado, 2015)

Dentro de la conciencia fonológica podemos encontrar diferentes niveles de complejidad. Según Herrera y Defior (2005) los niveles de conciencia fonológica son el nivel silábico, el de las unidades intrasilábicas (el ataque y la rima) y el fonémico.

Respecto a estos niveles podemos decir que cada uno de ellos requiere una diferente demanda cognitiva. El aprendizaje de un sistema alfabético ayuda al desarrollo de la conciencia fonémica, pero también hay otras habilidades que preceden a la adquisición de la lectura, como pueden ser la memoria verbal a corto plazo y la velocidad de denominación.

Numerosos autores consideran que la mejora de las habilidades fonológicas puede producir un mejor desarrollo de la lectura a corto plazo. (Márquez y de la Osa, 2003; Ripoll y Aguado, 2015)

Cuando diseñamos los programas para mejorar la conciencia fonológica de los niños/as es muy importante tener en cuenta los siguientes fundamentos (Ripoll y Aguado, 2015): los niños suelen acceder antes a la sílaba que al fonema; identificar es más fácil que manipular; omitir y añadir es más fácil que cambiar el orden o sustituir; las manipulaciones en la unidad inicial o final son más fáciles que las manipulaciones en las unidades intermedias; trabajar con palabras cortas es más fácil que con palabras largas; son más fáciles los fonemas que se pueden pronunciar independientemente y se pueden alargar, como las vocales y los fonemas /f/, /rr/ etc., que los que no se pueden pronunciar solos como /p/, /t/ o $/ \mathrm{k} /$;las imágenes o dibujos que representan a las palabras pueden facilitar las actividades y el uso de palabras técnicas como "silaba" o "fonema" pueden dificultar la comprensión de las actividades.

Para llegar a tener unas buenas habilidades fonológicas los alumnos/as deben ser capaces de realizar operaciones como las siguientes: identificar unidades iguales, añadir unidades, sustituir unidades y cambiar el orden de unidades. Dependiendo de si estas unidades son palabras, silabas o fonemas nos referiremos a conciencia de palabra, conciencia silábica o conciencia fonética.

Las dificultades de aprendizaje (DA) se refieren a un grupo heterogéneo de trastornos que, se manifiestan en problemas significativos en la adquisición y uso de las habilidades de recepción, habla, lectura, escritura, razonamiento, matemáticas, o problemas sociales relacionadas con la escuela. Estos trastornos son intrínsecos al individuo y se supone que son debidos a la disfunción del sistema nervioso central (Gómez et al., 2007).

En el aprendizaje de la lectura podemos encontrarnos con dos importantes dificultades que afectan al nivel léxico. Estos son el Retraso Lector y la Dislexia.

En cuanto al Retraso Lector, el alumnado que lo presenta suele tener un CI medio-bajo y dificultades en todas las áreas de aprendizaje. Estos niño/as aprenden a leer por un método que no es el adecuado o no están suficientemente estimulados. Sin embargo en la Dislexia presentan un CI medio-alto y las dificultades se encuentran solamente en las áreas en las que se utiliza la lectura.

Los lectores disléxicos y con retraso lector tiene un comportamiento similar respecto a la lectura ya que tienen problemas en la lectura de palabras y pseudopalabras.

En cuanto al retraso lector, si los lectores tienen fallos en la ruta fonológica o suelen confundir grafemas con rasgos fonológicos similares, hacen asimilaciones fonológicas $\mathrm{o}$ son incapaces de leer palabras desconocidas, podemos decir que se trata de un retraso lector disfonético. Sin embargo, cuando presentan fallos en la ruta visual, es decir, no poseen conciencia de palabra, cometen errores de omisión, adición, sustitución de letras etc. podemos decir que el retraso lector es diseidético.

Por su parte en la dislexia, podemos diferenciar entre la dislexia evolutiva y la adquirida. Las personas que presentan dislexia evolutiva tienen dificultades en la adquisición de la lectura desde su inicio. Sin embargo, las personas con dislexia adquirida se caracterizan por haber adquirido las habilidades lectoras adecuadas pero las han perdido a causa de una lesión cerebral.

En este último caso podemos diferenciar varios tipos de dislexia adquirida dependiendo de los errores que cometen en la lectura (Vieiro y Gómez, 2004).

\section{Objetivos}

El objetivo de este trabajo es la elaboración de materiales curriculares para el acceso a la lectura en base a un estudio de caso. 


\section{Método}

\section{Participantes}

Se ha aplicado un programa de mejora de la lectura a una niña de 10 años que presenta dificultades significativas en la lectura y en todas las demás áreas de aprendizaje. Con los datos obtenidos a través de su historial sabemos que su capacidad intelectual es media-baja según los resultados del WISC-IV, con un Cociente Intelectual Total de 85. En el momento de la intervención la niña cursaba $4^{\circ}$ de Primaria con una adaptación curricular en la que trabajaba los contenidos correspondientes al $2^{\circ}$ curso de Primaria. Además en el centro escolar también asistía a sesiones de Pedagogía Terapéutica y Audición y lenguaje.

Las sesiones de Pedagogía Terapéutica se realizaban una vez al día, bien dentro del aula ordinaria o en el aula de Pedagogía Terapéutica.

Las sesiones de Adición y Lenguaje se realizaban en el aula de Audición y Lenguaje y la duración de estas era de 40 minutos aproximadamente. Durante estas sesiones tuvo lugar la aplicación de este programa.

\section{Materiales}

Para realizar la evaluación tanto inicial como final se emplearon las pruebas que se describen a continuación:

-Prueba de Evaluación del Retraso en Lectura (P.E.R.E.L).

Es una prueba diseñada para la aplicación de forma individual a alumnos de $1^{\circ}$ a $3^{\circ}$ de Primaria.

Los objetivos de la prueba son los siguientes:

Obtener una escala en percentiles del rendimiento medio en lectura por parte del niño/a. A través de las tablas que nos ofrece podemos situar al niño en el percentil que le corresponde en función de su curso y trimestre escolar.

Evaluar el grado de retraso en la lectura. Las puntuaciones obtenidas se traducen a una escala que muestra la edad mental lectora en años y meses. Una vez obtenida la edad mental lectora estimada, se pasará a calcular el grado de retraso lector. Para ello se hace la resta entre la edad cronológica y la edad mental lectora del sujeto.

Identificar dificultades significativas en el aprendizaje de la lectura.

-Tareas de segmentación léxica, silábica y fonémica de Jiménez y Ortiz (1998)

Es una prueba que está compuesta por siete tareas entre las que se incluyen:

Segmentación léxica: Se presentan de forma oral las oraciones y el niño/a debe reconocer el número de palabras que contiene cada una de las oraciones. Para ello podrá ayudarse contando con los dedos, mediante palmadas o con tiras gráficas.

Aislar fonemas o sílabas en palabras: Se muestran una serie de dibujos en los cuales se debe buscar el sonido o sílaba que emite el examinador. Estos puede ser en posición inicial o final comenzando por los sonidos vocálicos, después las sílabas y finalmente los fonemas consonánticos.

Omisión de fonemas en palabras: esta actividad consiste en ir nombrando una serie de dibujos en los que se pide omitir primero el fonema vocálico inicial, después la sílaba inicial y finalmente la sílaba inicial.

Reconocer si la sílaba final, intermedia e inicial coincide con otra palabra: se presentan oralmente pares de palabras, primero bisílabas y después trisílabas, y el niño/a debe identificar primero si empiezan con la misma silaba y despues si terminan con la misma silaba.

Contar las sílabas de una palabra: se presentan las palabras oralmente y se pide que cuente las silabas de cada palabra pudiéndose ayudar con los dedos, con palmadas o con bloques manipulables.

Reconocer y pronunciar una palabra descompuesta en una secuencia de sílabas: la tarea consiste en reconocer una serie de palabras, que pueden ser bisílabas o trisílabas, descompuestas en sílabas, es decir, manteniendo un intervalo de tiempo entre cada sílaba al pronunciarlas.

Omisión de sílabas en palabras: se trata de nombrar una serie de dibujos omitiendo la sílaba que el examinador indica.

-Evaluación de los Procesos Lectores Prolec-Se (Ramos y Cuetos, 1999)

Es una prueba que evalúa los procesos cognitivos que intervienen en la lectura. Estos son: los procesos semánticos, los procesos sintácticos y el reconocimiento de palabras. Para ello utiliza seis pruebas diferentes: emparejamiento dibujo-oración, comprensión de textos, estructura de un texto, lectura de palabras, lectura de pseudopalabras y signos de puntuación.

Para realizar esta evaluación hemos empleado las subpruebas de lectura de palabras y lectura de pseudopalabras (Ramos y Cuetos, 1999)..

\section{Diseño}

Se trata de un trabajo de investigación constituido por un pretest, un tratamiento y un postest.

Las variables estudiadas fueron las propias medidas de los tests Prolec-se y P.E.R.E.L así como una prueba funcional de lectura en contexto.

\section{Procedimiento}

Posteriormente a la evaluación inicial se llevó a cabo un programa de intervención basado en la mejora de la conciencia fonológica y la discriminación visual. Las tareas de conciencia fonológica y discriminación visual se repartieron en 12 sesiones de una duración de 40 minutos cada una aproximadamente. Para estructurar la intervención se ha comenzado trabajando la conciencia fonológica. Esta se dividió en tres niveles empezando por la conciencia de palabra para luego desarrollar la conciencia silábica y finalmente el deletreo.

Para trabajar la discriminación visual espacial se comenzó con el uso de objetos, a continuación con figuras y finalmente con letras.

Las sesiones se han llevado a cabo de forma individual en el aula de audición y lenguaje de un colegio público de A Coruña en horario de mañana. Se han distribuido con una frecuencia de tres sesiones a la semana. El ambiente fue tranquilo y agradable. Antes de iniciar cada tarea se le explicó a la alumna el 
procedimiento y se le dio un ejemplo de cada una de las tareas. Para la elaboración de las actividades se procuró buscar frases sencillas con palabras de uso frecuente.

Las actividades que se realizaron para mejorar la conciencia fonológica fueron las siguientes:

Dentro de la conciencia de palabra se realizaron las siguientes actividades:

Suprimir palabras en una frase:

Se presentaron oralmente una serie de frases en las cuales la alumna debía identificar la última palabra para posteriormente repetir la misma frase pero omitiéndola.

Ejemplo:

$\begin{array}{lll}\text { Frase Juan } & \text { come gominolas } \\ \text { Respuesta } & \text { Juan come } \mathrm{X}\end{array}$

Contar palabras en una frase:

Para realizar esta actividad se empleó como apoyo visual un juego en el que se pega una ficha por cada palabra que tiene la frase.

\section{Añadir palabras a una frase:}

Esta tarea consiste en hacer frases más largas añadiéndole, de forma oral, palabras a una frase corta.

Ejemplo:

Nos gusta ir al parque.

Respuesta: - Nos gusta ir al parque para jugar.

Construir frases por número de palabras:

En esta actividad se le pide a la alumna que diga frases que tengan un número determinado de palabras.

Ejemplo:

Dime una frase que tenga cuatro palabras".

Respuesta: -Me gustan las galletas.

\section{Elegir la frase bien escrita:}

Esta tarea consiste en marcar la frase correcta entre dos o tres opciones. En las frases incorrectas el error consistía en falsas uniones de palabras o falsas separaciones.

Ejemplo:

"Marca la frase que está bien escrita".

Enel cine hay mu cha gente. En el cine hay mucha gente.

Separar las palabras en una frase:

Se presentan varias frases con las palabras unidas y la alumna debe separar dónde empieza y donde termina cada palabra para que la frase tenga sentido. Se ha empezado con frases sencillas y posteriormente se incrementó la dificultad utilizando frases más largas y poesías.

Ejemplo:

Esahabitaciónesmuybonita.

Esa/habitación/es/muy/bonita

Para mejorar la conciencia silábica se realizaron las siguientes actividades

Contar sílabas:
Para trabajar este aspecto se usaron fichas con dibujos para contar las sílabas.

Ejemplo:

“¿Cuál de los siguientes dibujos no tiene el mismo número de sílabas que los demás?”:

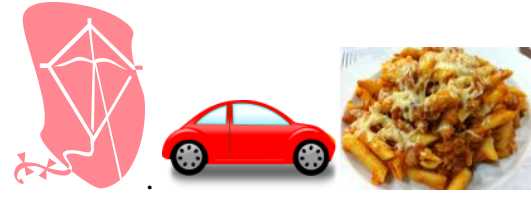

\section{Aislar sílabas:}

El objetivo de esta actividad fue identificar la sílaba inicial, intermedia y final a través de dibujos o juegos orales.

Las tareas realizadas consistieron en la omisión y adición de sílabas finales, iniciales e intermedias a través de dibujos o juegos orales.

Ejemplo:

-“Si a plátano le quitamos la última sílaba ¿qué palabra nos queda?":

$\begin{array}{lll}\text { Plá ta } & \text { no } \\ \text { Plá ta } & X\end{array}$

\section{Inversión silábica:}

Se trabajó la inversión de sílabas con apoyo visual a través de imágenes y también de forma oral. Se emplearon palabras bisílabas, las cuales formaban otra palabra al invertir sus sílabas.

Ejemplo:

"Si en la palabra casa cambiamos las silabas de orden, $\measuredangle$ qué palabra nos queda?"

$\mathrm{Ca} \mathrm{Sa}$

$\mathrm{Sa}$ ca

\section{Deletreo}

Para trabajar el deletreo se realizaron las siguientes actividades:

\section{Contar grafemas.}

Para realizar esta tarea se presentaron las palabras oralmente y se utilizó el apoyo visual mediante tiras gráficas para que la alumna marcase una cruz por cada letra que tenía la palabra.

Ejemplo:

“Cuántas letras tiene la palabra camisa?”

$\begin{array}{llllll}X & X & X & X & X & X\end{array}$

Aislar fonema inicial/aislar fonema final.

En esta actividad se le pidió a la alumna que repitiese una serie de palabras pero omitiendo el fonema inicial o final. 
Ejemplo:

"Si a foca le quitamos la $\mathrm{f}$ ¿qué palabra nos queda?"

Respuesta: Oca

Finalmente, se trabajaron tareas de discriminación vocálica a través de dibujos en los cuales se pedía marcar o pintar los que tuviesen un determinado fonema vocálico.

Ejemplo: "Colorea los dibujos que tienen en su nombre la letra que se indica"

En cuanto a la discriminación visual, se han trabajado tareas de orientación espacial con objetos, con símbolos y con letras. Para ello se realizaron durante dos sesiones las siguientes tareas:

-Discriminación visual espacial con objetos:

Se han empleado objetos cotidianos como cajas, bolígrafos, lápices o figuritas de animales para trabajar la discriminación espacial. Las actividades realizadas consistían en preguntas sobre la posición del objeto.

--Discriminación visual espacial con símbolos:

Para trabajar la orientación visual con símbolos, se llevó a cabo una tarea que consistía en identificar la figura idéntica al modelo. Para ello, tenía que rodear la figura colocada en la misma posición: hacia arriba, hacia abajo, mirando hacia la izquierda o mirando hacia la derecha.

Ejemplo: -"Busca las figuras que coinciden con la primera"

-Discriminación espacial con letras: Finalmente, se trabajó la discriminación de letras, utilizando letras visualmente similares (letras espejadas) como b/d, p/q y $\mathrm{u} / \mathrm{n}$ y también con vocales visualmente similares.

Ejemplo: -"Busca las letras que se indican en cada fila"

\section{Resultados}

Se ha realizado un análisis de los datos en función de los aciertos o los errores cometidos en cada prueba. A continuación se presentan los resultados obtenidos de las pruebas tanto en la evaluación inicial como en la evaluación final.

Para realizar la evaluación inicial hemos empleado las pruebas de Jiménez y Ortiz (1998), el P.E.R.E.L de Maldonado, Sebastián y Soto (1992) y finalmente la subprueba de lectura de pseudopalabras perteneciente al PROLEC-SE de Ramos y Cuetos (1999).

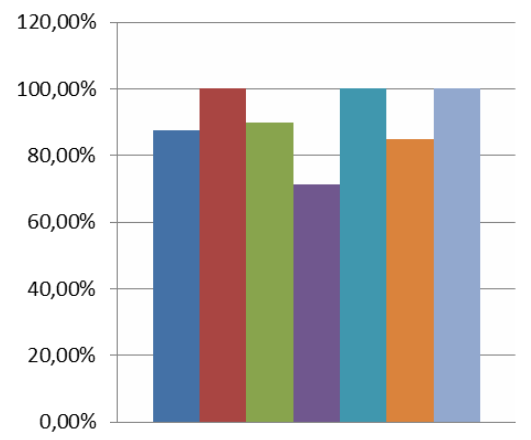

- Conciencia léxica

- Conciencia fonológica

- Contar sílabas

Eliminar sílabas

- Unir sílabas

Reconocer silabas

iguales

- Omisión de sílabas en palabras

Figura 1. Resultados pretest obtenidos en las diferentes tareas de segmentación léxica, silábica y fonémica de Jiménez y Ortiz (1998)

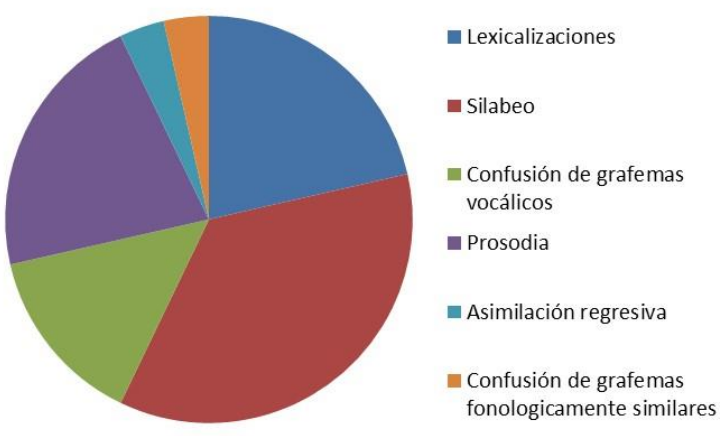

Figura 2. Resultados pretest obtenidos en la Prueba de Evaluación del Retraso Lector (P.E.R.E.L)

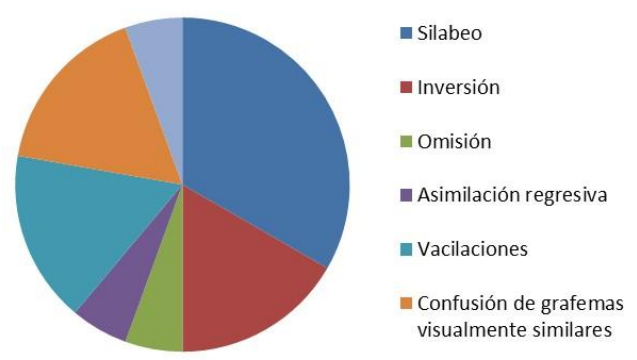

Figura 3. Resultados pretest obtenidos en la subprueba de lectura de pseudopalabras del Prolec-se

Tras la intervención se vuelven a evaluar los aspectos deficitarios. Y se comprueba que:

a) Mejoró su conciencia de palabra ya que no se producen lexicalizaciones en la evaluación final y disminuye también el silabeo.

b) Se aprecia una disminución en la confusión de grafemas visualmente similares y errores de omisión.

c) Desaparecen las asimilaciones regresivas, la confusión de grafemas fonológicamente similares y las vacilaciones, ya que no se aprecia ningún error de este tipo en la evaluación final.

Tabla 1. Porcentajes de errores en las distintas tareas

\begin{tabular}{lll}
\hline & Pretest & Postest \\
\hline Lexicalización & 10,7 & $\mathbf{0}$ \\
\hline Silabeo & 34,5 & $\mathbf{2 8 , 6}$ \\
\hline $\begin{array}{l}\text { Confusión de grafemas } \\
\text { vocálicos }\end{array}$ & 9,9 & 20,7 \\
\hline Errores de prosodia & 10,7 & 14 \\
\hline Asimilación regresiva & 4,6 & $\mathbf{0}$ \\
\hline $\begin{array}{l}\text { Confusión de grafemas } \\
\text { fonológicamente similares }\end{array}$ & 1,8 & $\mathbf{0}$ \\
\hline $\begin{array}{l}\text { Confusión de } \\
\text { graemas }\end{array}$ & 8,3 & $\mathbf{5 , 3}$ \\
\hline Adición intrasilábica & 0 & 2 \\
\hline Omisión & 2,78 & $\mathbf{2}$ \\
\hline Confusión “z” y “c” & 0 & 2 \\
\hline Inversión intrasilábica & 0 & 2 \\
\hline Vacilaciones & 8,3 & $\mathbf{0}$ \\
\hline Inversión de fonemas & 8,3 & 13,3 \\
\hline Confusión de /r/ y/rr/ & 0 & 10 \\
\hline
\end{tabular}


Sin embargo, sigue presentando confusión de grafemas vocálicos, errores de prosodia y de inversión de fonemas.

Además, tal y como podemos ver en la tabla 8 , en la evaluación final se detectaron algunos errores de adición intrasilábica, confusión de " $z$ " con "c", inversión intrasilábica.

\section{Conclusiones}

Después de haber aplicado este programa de intervención podemos decir que la niña mejoró a la hora de leer palabras desconocidas y redujo su nivel de lexicalizaciones. Esta mejoría la atribuimos a que se trabajó el deletreo con tareas como "contar grafemas" o "aislar el fonema inicial o final de cada palabra", presentando estas tareas de forma oral. La importancia del deletreo o del uso de la ruta fonológica para la lectura de palabras desconocidas está sustentado en estudios tales como los realizados por Bradley y Bryant (1985) en los que se aplicó un entrenamiento fonológico y de conocimiento de las letras a un grupo de niños y se ha demostrado que el conocimiento fonológico facilita el aprendizaje de la lectura y la escritura.

La mejoría en el silabeo la podemos atribuir al trabajo con actividades de conciencia de palabra tales como: "separar palabras en una frase", "contar las palabras que contiene cada frase" o "formar frases según el número de palabras". La importancia de la manipulación de las palabras para el desarrollo de la lectura está sustentado por autores como Calero, Pérez, Maldonado y Sebastian (1997) Daly, Neugebauer, Chafouleas y Skinner (2015). Interventions for reading problems: Designing and evaluating effective strategies. Guilford Publications.que defienden que la manipulación de las palabras ayuda al desarrollo de la conciencia fonológica y favorece el aprendizaje de la lectura.El objetivo principal de esta intervención fue el de conseguir una mejora en el desarrollo de la lectura.

Después de haber analizado los resultados podemos concluir que, tras la intervención, la niña mejoró su capacidad de deletreo cometiendo menos lexicalizaciones.

También podemos observar una mejora en su lectura global, ya que se redujo el silabeo, aunque no ha logrado un uso perfecto de la ruta visual, lo que implica que en la prosodia siga cometiendo errores.

No presenta mejora en actividades de discriminación visual espacial que puede ser debido al hecho de que solo se dedicó la última semana de la intervención a las tareas de este tipo.

Sigue presentando errores de conciencia silábica relacionados con la memoria de trabajo. A pesar de haber realizado casi todas las actividades en leguaje oral, sigue presentando errores de carácter ejecutivo.

Con respecto a la confusión de grafemas, sigue presentando dificultades con las vocales pero ha mejorado en cuanto a los grafemas visualmente similares y los grafemas fonológicamente similares, no cometiendo tantos errores en estos.

\section{Referencias bibliográficas}

Bryant, P. y Bradley, L. (1985). Problemas Infantiles de lectura. Madrid: Alianza.

Calero, A., Pérez, R., Maldonado, A. y Sebastian, E. (1997). Materiales para favorecer el acceso a la lectura en Educación Infantil. Madrid: Escuela Española

Cuetos, F., Rodríguez, B., Ruano, E. y Arribas, D. (2014). PROLEC-R. Batería de Evaluación de los Procesos Lectores, Revisada (5 ${ }^{a}$ Edición). Madrid: TEA Ediciones.

Daly, E. J., Neugebauer, S., Chafouleas, S. M., \& Skinner, C. H. (2015). Interventions for reading problems: Designing and evaluating effective strategies. Guilford Publications.

García, E. (1993). La comprensión de textos. Modelo de procesamiento y estrategias de mejora. Didáctica.5, 87-113.

Gómez, L.A., Duarte, A.M., Merchán, V., Aguirre, D.C. y Pineda, D. (2007). Conciencia fonológica y comportamiento verbal en niños con dificultades de aprendizaje. Universitas Psychologica, 6 (3), 571-580.

Guzmán R. (1997). Métodos de lectura y acceso al léxico (Tesis doctoral). Universidad de la Laguna, Tenerife, Islas Canarias.

Herrera, L. y Defior, S. (2005). Una aproximación al procesamiento fonológico de los niños prelectores: Conciencia fonológica, memoria verbal a corto plazo y denominación. Psykhé, 14 (2), 81-95.

Jiménez, J. E. (1992). Metaconocimiento fonológico: estudio descriptivo sobre una muestra de niños prelectores en edad preescolar. Infancia $y$ Aprendizaje,57, 49-66.

Jiménez, J. E y Ortiz, M. (2001). Conciencia fonológica y aprendizaje de la lectura: Teoría, evaluación e intervención. Madrid: Sintesis.

Márquez, J., y de la Osa, P. (2003). Evaluación de la conciencia fonológica en el inicio lector. Anuario de Psicología, 34 (3), 357-370.

Ripoll, J.C. y Aguado, G. (2015). Enseñar a leer. Cómo hacer lectores competentes. Madrid: Eos.

Soto, P., Maldonado, A. y Sebastián, E. (1992). Retraso en lectura: evaluación y tratamiento educativo. Madrid: UAM.

Vieiro, P., Peralbo, M. y García, J.A. (1997). Procesos de adquisición y producción de la lectoescritura. Madrid: Visor.

Vieiro, P. y Gomez, I. (2004). Psicología de la lectura. Madrid: Pearson. 